\title{
DETERMINANTAL INEQUALITIES FOR
}

\section{BLOCK TRIANGULAR MATRICES}

\author{
MINGHUA LIN
}

Abstract. Let $T=\left[\begin{array}{ll}X & Y \\ 0 & Z\end{array}\right]$ be an $n$-square matrix, where $X, Z$ are $r$-square and $(n-r)$-square, respectively. Among other determinantal inequalities, it is proved that

$$
\operatorname{det}\left(I_{n}+T^{*} T\right) \geqslant \operatorname{det}\left(I_{r}+X^{*} X\right) \cdot \operatorname{det}\left(I_{n-r}+Z^{*} Z\right)
$$

with equality if and only if $Y=0$.

Mathematics subject classification (2010): 15A45.

Keywords and phrases: Determinantal inequality, block triangular matrices.

\section{REFERENCES}

[1] D. Z. Duoković, On some representations of matrices, Linear Multilinear Algebra 4 (1976) 33-40.

[2] D. Z. Duoković, O. P. Lossers, Problem E2525, $\operatorname{det}(I+A \bar{A}) \geqslant 0$, Amer. Math. Monthly 83 (1976) 483-484.

[3] S. W. DRURY, The canonical correlations of a $2 \times 2$ block matrix with given eigenvalues, Linear Algebra Appl. 354 (2002) 103-117.

[4] R. A. Horn, C. R. Johnson, Matrix Analysis, Cambridge University Press, 2nd ed., 2013.

[5] R. A. Horn, C. R. Johnson, Topics in Matrix Analysis, Cambridge University Press, 1991.

[6] A. W. Marshall, I. Olkin, B. Arnold, Inequalities: Theory of Majorization and Its Applications, Springer, New York, 2nd ed., 2011. 